\title{
Cultured human amniocytes express hTERT, which is distributed between nucleus and cytoplasm and is secreted in extracellular vesicles
}

\author{
Annalisa Radeghieri" ${ }^{*}$, , Giulia Savio ${ }^{1}$, Andrea Zendrini, Giuseppe Di Noto, \\ Alessandro Salvi, Paolo Bergese, Giovanna Piovani ${ }^{* *}$ \\ Department of Molecular and Translational Medicine, University of Brescia, Italy, Viale Europa 11, 25123, Brescia, Italy
}

\section{A R T I C L E I N F O}

\section{Article history:}

Received 18 November 2016

Accepted 11 December 2016

Available online $\mathrm{xxx}$

\section{Keywords:}

Amniotic fluid cells

hTERT

Gene expression

Intracellular distribution

Extracellular vesicles

\begin{abstract}
A B S T R A C T
Background: An increasing number of studies on stem cells suggests that the therapeutic effect they exert is primarily mediated by a paracrine regulation through extracellular vesicles (EVs) giving solid grounds for stem cell EVs to be exploited as agents for treating diseases or for restoring damaged tissues and organs.

Due to their capacity to differentiate in all embryonic germ layers, amniotic fluid stem cells (AFCs), represent a highly promising cell type for tissue regeneration, which however is still poorly studied and in turn underutilized. In view of this, we conducted a first investigation on the expression of human hTERT gene - known to be among the key triggers of organ regeneration - in AFCs and in the EVs they secrete.

Methods: Isolated AFCs were evaluated by RT-qPCR for hTERT expression. The clones expressing the highest levels of transcript, were analyzed by Immunofluorescence imaging and Nuclear/cytoplasmic fractionation in order to evaluate hTERT subcellular localization. We then separated EVs from FBS depleted culture medium by serial (ultra) centrifugations steps and characterized them using Western blotting, Atomic force Microscopy and Nanoplasmonic assay.

Results: We first demonstrated that primary cultures of AFCs express the gene hTERT at different levels. Then we evidenced that in AFCs with the higher transcript levels, the hTERT protein is present in the nuclear and cytoplasmic compartment. Finally, we found that cytosolic hTERT is embodied in the EVs that AFCs secrete in the extracellular milieu.

Conclusions: Our study demonstrates for the first time the expression of the full protein hTERT by AFCs and its release outside the cell mediated by EVs, indicating a new extra telomeric role for this protein. This finding represents an initial but crucial evidence for considering AFCs derived EVs as new potential sources for tissue regeneration.
\end{abstract}

() 2016 Elsevier Inc. All rights reserved.

\section{Introduction}

The rapid advances in the field of regenerative medicine have identified novel sources of stem cells with potential for therapy.

\footnotetext{
Abbreviations: SC, Stem Cells; AFCs, Amniotic fluid cells; EVs, Extracellular vesicles.

* Corresponding author.

** Corresponding author.

E-mail addresses: annalisa.radeghieri@unibs.it (A. Radeghieri), giovanna. piovani@unibs.it (G. Piovani).

${ }^{1}$ These authors contributed equally to the experimental of this work.
}

Recently, the amniotic fluid (AF) has been acknowledged as an important and alternative, yet underutilized source of cells [1]. AF cells (AFCs) represent a heterogeneous population composed of three groups of adherent cells, categorized based on their morphological, proliferative and biochemical characteristics: epithelioid (E-type), amniotic fluid (AF-type) and fibroblastic (Ftype) cells [2]. In addition to fully differentiated cells, precursors and multipotent stem like cells have been described within AF [3]. Those cells show high plasticity and the capacity to differentiate into the three types of germ layer cells, making them ideal for applications in regenerative medicine [4], eventually banked and used for treatments. 
However, the effective clinical translation of AFCs based approaches requires in vitro culture expansion to achieve a sufficient number of cells and strict characterization of the cultures, two factors that may severely hamper their clinical use [5].

In addition, very recent data indicate that the structural contribution of stem cells (SCs) to regenerated tissues seems to be limited, and that rather the stimulation of local healing processes plays an important role in regeneration [6]. This observation prompted research to focus on the paracrine hypothesis, investigating the stimulating factors released by SCs, including growth factors and cytokines, often delivered by extracellular vesicles (EVs) $[7,8]$. Indeed, EVs derived from mesenchymal SCs have the ability to induce tissue regeneration by delivering growth factors, proteins, miRNA, mRNA, non coding RNA, and lipids [9]. Several reports have demonstrated the feasibility to regenerate tissues and neovascularization in models of myocardial infarction, skeletal muscle and kidney injury using exosomes from SCs and endothelial progenitor cells [10-12]. Thus it is feasible that, in the future, SC derived EVs could be used instead of SC to treat various diseases [13].

The ability of a cell to proliferate is an essential feature in the regeneration process and is tightly linked to its ability to maintain healthy telomeres and avoid DNA damage during DNA replication.

Telomerase is a ribonucleoprotein enzyme that maintains telomere length by adding DNA repeats to chromosome ends [14]. Because of its ability to counteract telomere erosion, telomerase is associated with the potential proliferation of cells and tissues [15] and has been implicated in mouse liver regeneration [16] and zebrafish heart regeneration [17].

The hTERT protein is the catalytic subunit of telomerase and plays a fundamental role during foetal growth. Its ectopic expression in normal human cells confers telomerase activity and stabilizes telomere lengths. hTERT expression is stringently regulated in most adult somatic tissues, which commonly, express no or very low levels of hTERT mRNA [18] with several exceptions [19].

In the current study, we aimed to characterize the expression of hTERT in AFCs, based on the capacity of these cells to differentiate in SCs, and the possibility that AFCs secrete the protein via EVs. Currently, no data are available on hTERT expression and localization in these cells. Furthermore, no research yet studied if telomerase positive SCs could influence telomerase negative somatic wounded cells via EV crosstalk. In a related way, a very recent paper showed that hTERT mRNA contained in exosomes derived from tumor cells is able to transform non malignant fibroblasts into telomerase positive cells [20], increasing proliferation, life span and protecting them from DNA damage.

Our results describe for the first time hTERT expression in AFCs and that the whole protein is included in EVs derived from AFCs. This data represents interesting evidence towards understanding the promising therapeutic potential of these cells in regenerative medicine, suggesting a possible mechanism by which those cells could exert a positive effect towards neighboring wounded tissues, which will be explored in the future.

\section{Materials and methods}

\subsection{Cells cultures and hTERT gene asset}

The study on amniotic fluid was performed on 25 samples (12 were $46, \mathrm{XY}$ and 13 were $46, \mathrm{XX}$ ), established following amniocentesis. A small aliquot of cells was obtained after the analysis and the results of prenatal diagnosis. Written informed consents to use the cells, only for research purposes, were obtained from the patients. All samples were rendered anonymous and stored, at passage 1 , with the concentration of $1 * 10^{6} / \mathrm{ml}$ in cryogenic system of our department. After thawing cells were cultured in $100 \mathrm{~mm}$ tissue culture Petri (Jet Biofil) at $37{ }^{\circ} \mathrm{C}, 5 \% \mathrm{CO}_{2}$, with DMEM (Carlo Erba) supplemented with 20\% FBS 1\% glutamine, 1\% antibiotics (Gibco).

Copy number variation (CNV) of $h T E R T$ was used to evaluate the number of $h T E R T$ gene copies to avoid the presence of cryptic rearrangements. We used the following primers: TaqMan Copy Number Reference Assay RNAse P and TaqMan Copy Number Assay hs00233340 (exon 1 TERT) e hs01237576 (exon 16 TERT) (Applied Biosystem).

Data processing was evaluated by software Copy Caller 1.0 (Applied Biosystem).

\subsection{Gene expression}

The total RNA from amniocytes samples was isolated using TRIzol reagent (Invitrogen), according to the manufacturer's instructions and was transcribed into cDNA using random primers, ribonuclease inhibitor and M-MLV reverse trascriptase (Invitrogen)

The expression of hTERT mRNA in the samples was evaluated using TaqMan Gene Expression Assay (hs00972656 for hTERT) (Applied Biosystem). GAPDH was used as an internal standard.

The expression of hTERT mRNA [17] was based on the $\Delta \Delta \mathrm{Ct}$ method.

\subsection{Immunofluorescence (IF) imaging}

AFCs were cultured on $11 \mathrm{~mm}$ glass coverslips until $60-80 \%$ confluence. IF was performed following primary antibody datasheet instructions (rabbit anti-hTERT, Rockland Immunochemicals Inc.). Confocal microscopy was performed on a ZEISS LSM 510 META confocal laser scanning microscope using the $63 \times$ or the $100 \times$ Plan-NEOFLUAR oil immersion objective. Single confocal sections are shown for each condition. Images were processed with the use of Image $\mathrm{J}$ [21].

\subsection{Nuclear/cytoplasmic fractionation}

Fractionation of AFCs was performed as described [22]. Whole cell homogenate, cytosolic and nuclear fractions were boiled and loaded on a SDS-PAGE as described below.

\subsection{Western-blot analysis}

SDS sample buffer was added to isolated EVs or cellular extracts and samples were boiled $5 \mathrm{~min}$ at $95{ }^{\circ} \mathrm{C}$. Samples were electrophoresed in SDS-PAGE 10\% and analyzed by Western-blot (WB) with rabbit anti-hTERT (Rockland Immunochemicals Inc.) [23], mouse anti-Hsp 70 (Enzo Life Science), mouse anti-CD63 (Merck), mouse anti-Annexin-V (Santa Cruz) and/or mouse anti-Lamin A/C (Thermofisher) antibodies.

\subsection{EV separation and characterization}

Cell culture media from $5^{*} 10^{7}$ cultured cells, grown in the presence of $10 \%$ vesicle depleted FBS, was processed with serial centrifugation steps as previously described [24]. Pellets were resuspended in $50 \mu \mathrm{L}$ PBS $1 \times$ supplemented with 1:1000 Protease Inhibitor Cocktail (Sigma).

EV preparations were checked for purity by adapting the colorimetric nanoplasmonic assay we previously developed [25].

For AFM imaging, EV preparations were diluted 1:10 with deionized water. Five to $10 \mu \mathrm{L}$ of samples were then spotted onto freshly cleaved mica sheets (Grade V-1, thickness $0.15 \mathrm{~mm}$, size $10 \times 10 \mathrm{~mm}$ ). All mica substrates were dried at RT and analyzed 
using a Nanosurf NaioAFM (Nanosurf AG), equipped with Tap190AI-G tips (Budget Sensors). Images were snapped in tapping mode; the scan size ranged from $0.5 \mu \mathrm{m}$ to $15 \mu \mathrm{m}$; the scan speed ranged from 0.6 to $1.5 \mathrm{~s} x$ line.

EV size distribution was obtained by image analysis of three representative AFM images of scan size $5 \times 5 \mu \mathrm{m}$ performed by the WSxM 5.0 software (http://www.wsxmsolutions.com).

EV biochemical characterization was performed by Western blot as described above.

\section{Results and discussion}

\section{1. hTERT gene is present in a diploid asset in AFCs but the expression is different among samples}

In order to evaluate hTERT gene expression in AFCs we used frozen samples stored in our cell bank. After thawing, all samples of AFCs demonstrated the capacity to grow and triplicated the growth within 14 days, in according with literature [5]. All experiments were performed at passage 3 on the heterogeneous cellular population obtained by expansion of the samples after thawing. We deliberately planned to investigate this population of AFCs because cells are generally frozen and banked in this heterogeneous condition. If necessary, they might be differentiated towards mesenchymal stem cells or the three types of germ layer cells.

We evaluated the diploid asset of hTERT gene by genomic DNA CNV analysis, confirming that in all samples the hTERT is present in two copies (data not shown). Gene expression studies showed heterogeneous expression levels for hTERT gene among samples (Fig. 1). The gene is expressed in 18/25 samples analyzed (72\%) with a $R Q$ value ( $R Q=2^{-\Delta \Delta C t}$ ) comprised between 1 and 11.8 . The detection and the variability of expression values suggest the hypothesis that they are probably correlated to the presence of different cell types in the AF samples as previously described [4].

\section{2. hTERT protein is expressed in amniocytes and is partitioned between nuclei and cytoplasm}

hTERT protein expression is regulated by a complex system, predominantly involving transcriptional and translational control. It has been shown that the subcellular location of hTERT is dynamic and dependent on the cell cycle, DNA damage or cellular transformation [26]. Notably, a number of studies have shown that hTERT, which is regarded as a nuclear protein, has been identified

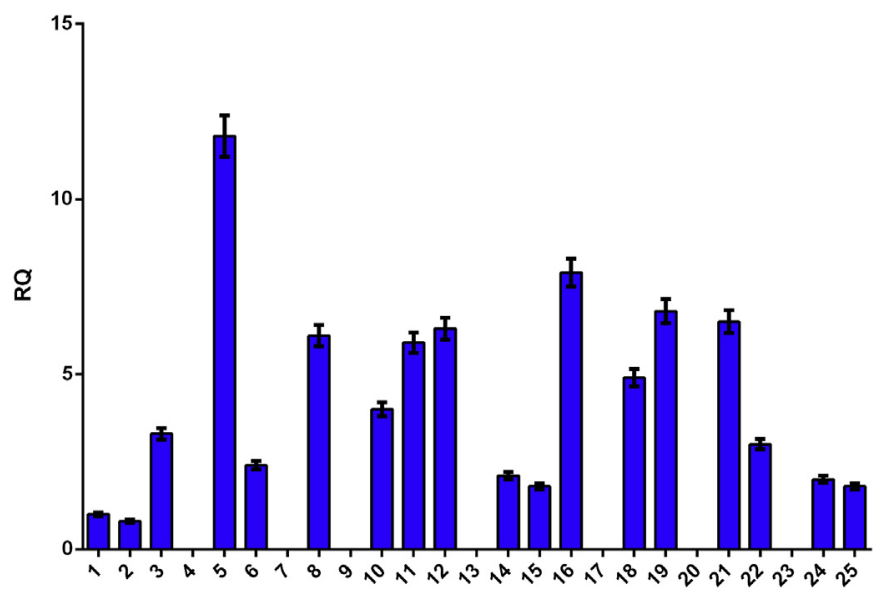

Fig. 1. hTERT Gene Expression. ( $\left.R Q=2^{-\Delta \Delta \mathrm{ct}}\right)$. The gene is expressed in 18 of samples analyzed with a $R Q$ value $\left(R Q=2^{-\Delta \Delta C t}\right)$ comprised between 1 and 11.8 . not only in the nucleus but also occasionally in the cytoplasm of different tumor cell lines and peripheral blood cells. In addition, it is subjected to CRM-1 dependent nucleocytoplasmic trafficking [27]. hTERT subcellular localization remains however a controversial issue [28]. Since hTERT protein expression has never been shown in amniocytes before, we wanted to visualize its presence and subcellular localization in selected AFCs, which showed a good gene expression pattern (RQ value above 4,8 samples). We separated the nuclear fraction from the cytosolic pool as indicated in Materials and methods. As shown in Fig. 2A, hTERT protein is mainly concentrated in the nucleus of the cells, but the presence of the protein is also detectable in the cytosolic fraction. Immunofluorescence (IF) staining performed on the same AFCs grown on glass coverslips also corroborates this result. Cells were fixed, permeabilized and analyzed using the rabbit anti-hTERT antibody. Fig. 2B shows a specific staining of the protein in the nucleus, especially in nucleoli, and in the cytoplasm, in reticulated like structures. Furthermore, it is to notice that the staining is different among the cell population. Actually, some cells have a low nucleolar staining but a strong non dispersed cytosolic signal, possibly related to filaments of actin or tubulin. This distribution appears in accordance with literature data, showing that knockdown of hTERT alters the biophysical properties of cells leading to reorganization of the cytoskeleton [29].

Thus we can conclude that in AFCs the protein hTERT is expressed and its subcellular localization is not only confined to nuclei but can be dispersed throughout the cytosol, meaning that in those cells hTERT might play different functions [30] and might be directed to different routes. hTERT distribution shows a variability among AFCs considered, which might be due to single cell conditions, cell cycle, or cell type considered.

\section{3. hTERT protein is embodied in extracellular vesicles secreted by AFCs}

EVs were isolated from cell culture medium of AFCs obtained from different amniotic fluids selected for normal diploid karyotype and $h T E R T$ RQ value above 4 . AFCs were cultured in DMEM medium supplemented with vesicles depleted FBS. EVs were purified by means of serial centrifugations to eliminate cellular debris and big protein aggregates from $40 \mathrm{~mL}$ of cell medium obtained from $90 \%$ confluent T75 flasks.

We first assessed the purity of EV samples by applying a colorimetric nanoplasmonic assay, which exploits the colloidal and optical properties of gold nanoparticle (AuNPs) solutions [25]. Briefly, bare AuNPs adsorb and cluster at the EV membrane in pure preparations. Instead, if the preparations contain exogenous contaminants, AuNPs are preferentially coated by those contaminants, which prevent AuNPs from clustering. Aggregation drives a red shift of the localized surface plasmon resonance (LSPR) absorption peak of the AuNPs (Fig. S1), leading the solution to a red to blue color change proportional to the purity grade of the added EV preparation.

AuNPs clustering can be quantified by the Aggregation Index $(A I)$, which directly relates the AuNP aggregation with preparation purity grade. AI can be determined from the sample UV/Vis spectra and is here conveniently defined as the ratio between the $519 \mathrm{~nm}$ and $650 \mathrm{~nm}$ LSPR absorbance $\left(A I=A_{519} / A_{650}\right)$. $A I$ decreases along with the change of the solution color from red to blue, and is inversely proportional to the preparation purity. The determined $A I$ for our samples is around 2.58 (Fig. 3A), close to the reference value of the pure AuNP solution, corresponding to a high purity level.

The morphological properties of the vesicles and their size distribution were determined by means of atomic force microscopy (AFM). EV preparation was adsorbed on a freshly cleaved mica 
A

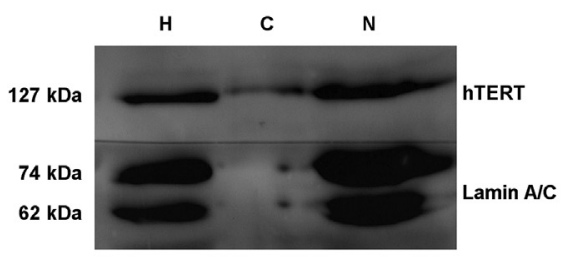

B
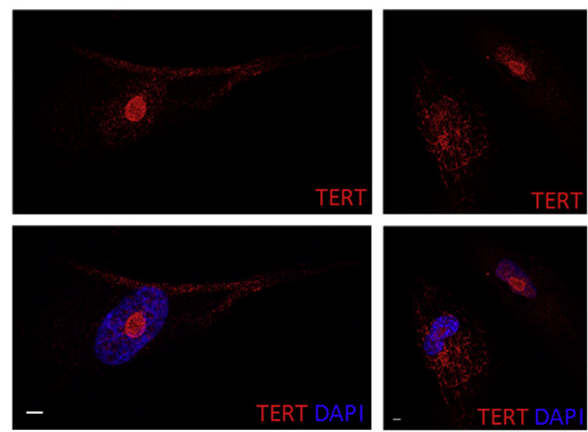

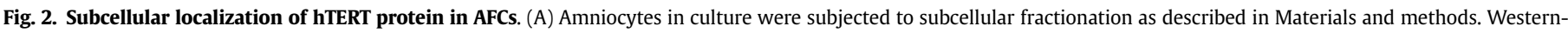

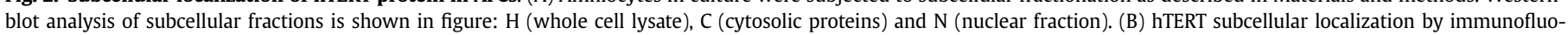
rescence analysis. Amnyocites were fixed with PFA 4\%, permeabilized with NP-40 0.2\% and stained with rabbit anti-hTERT antibody. Scale bars, $5 \mu$ m.

A

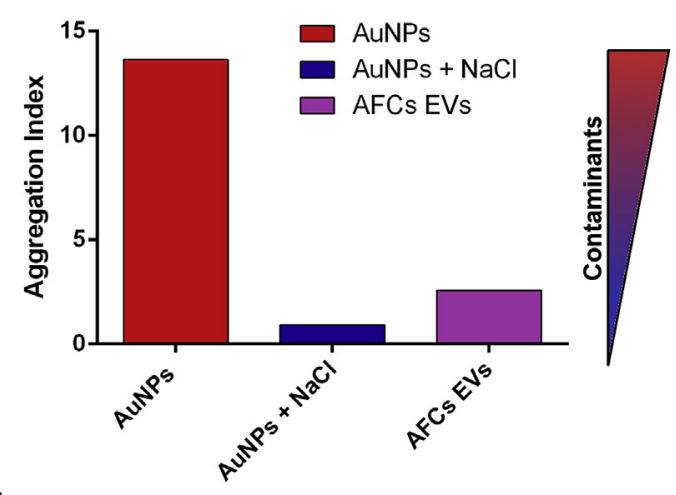

C

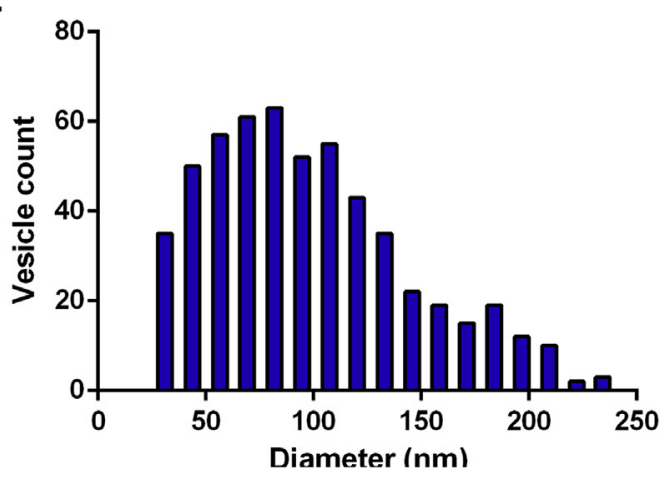

B

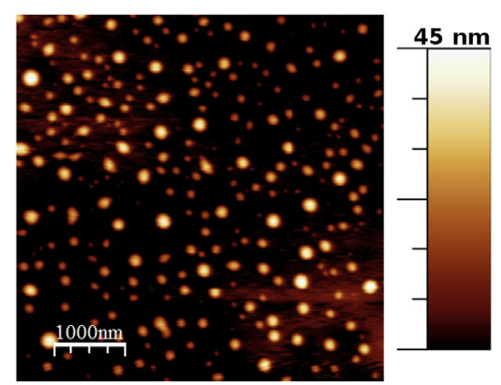

D

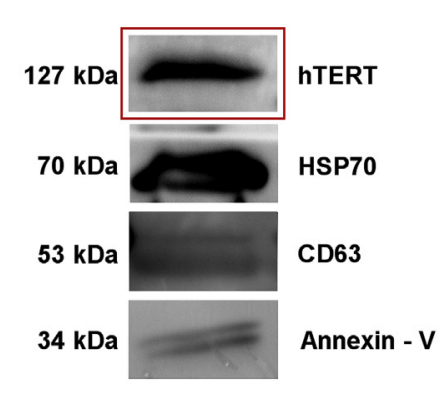

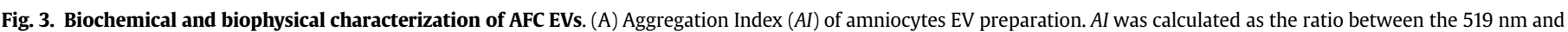

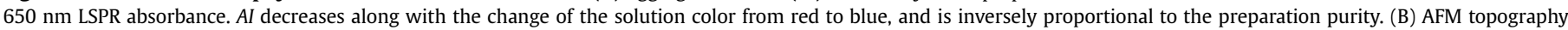

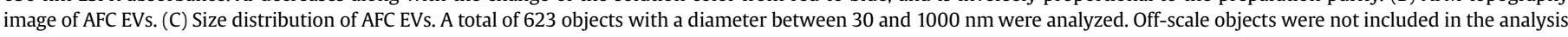

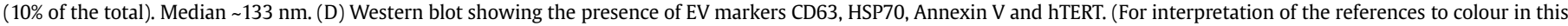
figure legend, the reader is referred to the web version of this article.)

surface and imaged in ambient condition. The representative image displayed in Fig. 3B shows the imaged portion of the sample is composed by EVs with a size ranging from tens to few hundreds of $\mathrm{nm}$. The black background indicates the absence of any relevant nanoscale feature other than the EVs (with reference to the color code bar), confirming the absence of residual contaminants from the separation procedure and the purity of the sample [31]. Image analysis allowed to quantify and plot the size distribution of the EVs, which is reported in bar chart form in Fig. 3C. Remarkably, the distribution is asymmetrical, ranging from $30 \mathrm{~nm}$ to $240 \mathrm{~nm}$ peaking at the size of $60-70 \mathrm{~nm}$. This observation indicates that AFCs EV population is prevalently composed by EVs with a size below $100 \mathrm{~nm}$, which is the typical size of exosomes.

We then performed a biochemical characterization of the samples by Western Blot analysis using the conventional EV markers HSP70, CD63 and Annexin-V, which we all found expressed in our vesicles. Through Western Blotting, we also discovered for the first time the presence of hTERT in those samples, showing a clear band at around $130 \mathrm{kDa}$ (Fig. 3D).

As it has been documented before [32], exosomes are small EVs 


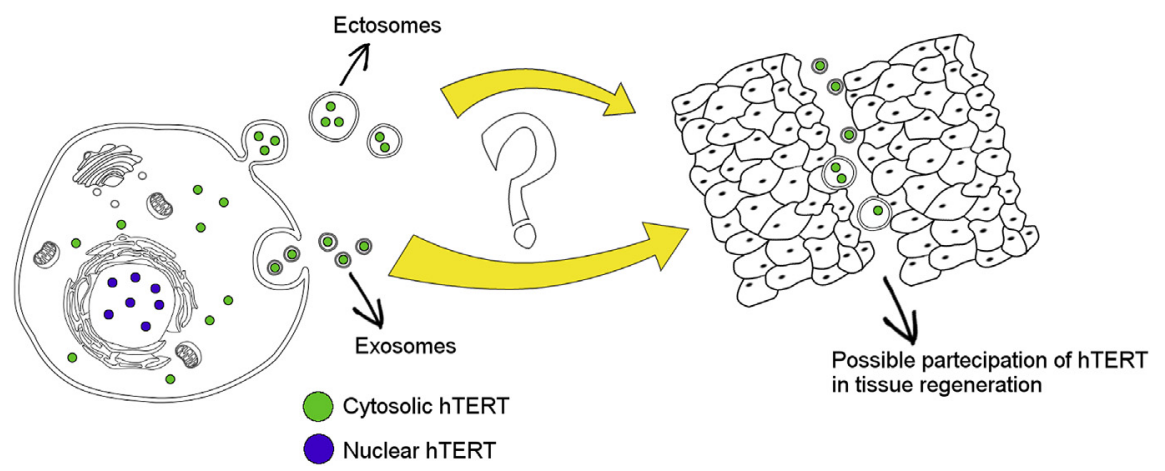

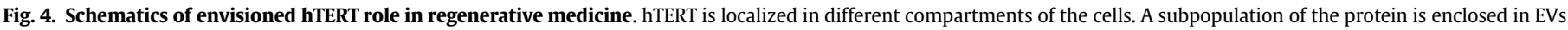

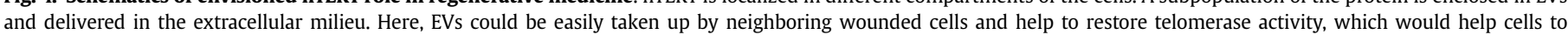
proliferate and heal the tissue.

released from cells upon fusion of an intermediate endocytic compartment, the multivesicular body (MVB). As a result of an engulfment of cytosolic material they contain several cytosolic proteins. By our biochemical and biophysical analyses we can speculate that the main population of vesicles secreted by AFCs is composed of exosomes. Since we found that hTERT is also localized in the cytoplasmic compartment, we suppose that "gulps" of cytosol containing hTERT are enclosed in intraluminal vesicles in MVB, which are then released.

It has recently been demonstrated that hTERT mRNA is delivered via exosomes to fibroblasts and modulates telomerase activity on these cells [20]. To this finding we add that the whole ready to go protein is enclosed in AFCs derived exosomes, suggesting that the effect previously described might be induced by the protein transferred itself and not only by mRNA transfer. This could mean a quicker activation of the processes described.

It could be also possible that a portion of cytosolic hTERT might be freely released in the extracellular milieu: we deliberately did not investigate this hypothesis since it seems unlikely that the protein could be endocytosed by cells as a functional molecule. Furthermore, the transfer of functional biological molecules by EVs is being more and more unveiled together with the effect exerted by EVs towards target cells [33], as we envision for hTERT in Fig. 4. This finding, if verified on AFCs towards somatic wounded cells (experimental work has been started), could open the route to studies for the employment of AFCs in healing tissues and regenerative medicine developing new avenues for future research.

\section{Acknowledgements}

We would like to thank Doris Ricotta, who introduced us to EV research, for fruitful discussions and sharing ideas and perspectives. We would like to thank Eugenio Monti and Lucia Paolini for critical reading of the manuscript and Luigi Caimi for his constant support.

This work was supported by University of Brescia research fund (RIC_ATENEO_13_RADEGHI_7_EX60, 2014_PIOVANI_EX60\%). G.D.N. received a fellowship "Bando Mecenate" (Borsa di Studio Bando Mecenate 2015) from "Fondazione CEUR", Bologna (Italy).

\section{Transparency document}

Transparency document related to this article can be found online at http://dx.doi.org/10.1016/j.bbrc.2016.12.077.

\section{Appendix A. Supplementary data}

Supplementary data related to this chapter can be found at http://dx.doi.org/10.1016/j.bbrc.2016.12.077.

\section{References}

[1] S.P. Loukogeorgakis, P. De Coppi, Stem cells from amniotic fluid-Potential for regenerative medicine, Best. Pract. Res. Clin. Obstet. Gynaecol. 31 (2016) $45-57$.

[2] H. Hoehn, E.M. Bryant, A.G. Fantel, G.M. Martin, Cultivated cells from diagnostic amniocentesis in second trimester pregnancies. III. The fetal urine as a potential source of clonable cells, Humangenetik 29 (1975) 285-290.

[3] O. Parolini, M. Soncini, M. Evangelista, D. Schmidt, Amniotic membrane and amniotic fluid-derived cells: potential tools for regenerative medicine? Regen. Med. 4 (2009) 275-291.

[4] M. Cananzi, P. De Coppi, CD117(+) amniotic fluid stem cells: state of the art and future perspectives, Organogenesis 8 (2012) 77-88.

[5] F. Iordache, A. Constantinescu, E. Andrei, B. Amuzescu, F. Halitzchi, L. Savu, H. Maniu, Electrophysiology, immunophenotype, and gene expression characterization of senescent and cryopreserved human amniotic fluid stem cells, J. Physiol. Sci. 66 (2016) 463-476.

[6] O.G. De Jong, B.W. Van Balkom, R.M. Schiffelers, C.V. Bouten, M.C. Verhaar, Extracellular vesicles: potential roles in regenerative medicine, Front. Immunol. 5 (2014) 608.

[7] J.G. Patton, J.L. Franklin, A.M. Weaver, K. Vickers, B. Zhang, R.J. Coffey, K.M. Ansel, R. Blelloch, A. Goga, B. Huang, N. L'Etoille, R.L. Raffai, C.P. Lai, A.M. Krichevsky, B. Mateescu, V.J. Greiner, C. Hunter, O. Voinnet, M.T. McManus, Biogenesis, delivery, and function of extracellular RNA, J. Extracell. Vesicles 4 (2015) 27494.

[8] T. Katsuda, N. Kosaka, F. Takeshita, T. Ochiya, The therapeutic potential of mesenchymal stem cell-derived extracellular vesicles, Proteomics 13 (2013).

[9] S. Fais, L. O'Driscoll, F.E. Borras, E. Buzas, G. Camussi, F. Cappello, J. Carvalho, A. Cordeiro da Silva, H. Del Portillo, S. El Andaloussi, T. Ficko Trcek, R. Furlan, A. Hendrix, I. Gursel, V. Kralj-Iglic, B. Kaeffer, M. Kosanovic, M.E. Lekka, G. Lipps, M. Logozzi, A. Marcilla, M. Sammar, A. Llorente, I. Nazarenko, C. Oliveira, G. Pocsfalvi, L. Rajendran, G. Raposo, E. Rohde, P. Siljander, G. van Niel, M.H. Vasconcelos, M. Yanez-Mo, M.L. Yliperttula, N. Zarovni, A.B. Zavec, B. Giebel, Evidence-based clinical use of nanoscale extracellular vesicles in nanomedicine, ACS Nano 10 (2016) 3886-3899.

[10] A. Dorronsoro, P.D. Robbins, Regenerating the injured kidney with human umbilical cord mesenchymal stem cell-derived exosomes, Stem Cell Res. Ther. 4 (2013) 39.

[11] R.C. Lai, F. Arslan, M.M. Lee, N.S. Sze, A. Choo, T.S. Chen, M. Salto-Tellez, L. Timmers, C.N. Lee, R.M. El Oakley, G. Pasterkamp, D.P. de Kleijn, S.K. Lim, Exosome secreted by MSC reduces myocardial ischemia/reperfusion injury, Stem Cell Res. 4 (2010) 214-222.

[12] Y. Nakamura, S. Miyaki, H. Ishitobi, S. Matsuyama, T. Nakasa, N. Kamei, T. Akimoto, Y. Higashi, M. Ochi, Mesenchymal-stem-cell-derived exosomes accelerate skeletal muscle regeneration, FEBS Lett. 589 (2015) 1257-1265.

[13] S. Ohno, G.P. Drummen, M. Kuroda, Focus on extracellular vesicles: development of extracellular vesicle-based therapeutic systems, Int. J. Mol. Sci. 17 (2016) 172.

[14] C.W. Greider, E.H. Blackburn, Identification of a specific telomere terminal transferase activity in tetrahymena extracts, Cell 43 (1985) 405-413.

[15] I. Flores, M.A. Blasco, The role of telomeres and telomerase in stem cell aging, FEBS Lett. 584 (2010) 3826-3830.

[16] S. Tumpel, K.L. Rudolph, The role of telomere shortening in somatic stem cells and tissue aging: lessons from telomerase model systems, Ann. N. Y. Acad. Sci. 
1266 (2012) 28-39.

[17] D. Bednarek, Juan M. González-Rosa, G. Guzmán-Martínez, Ó. GutiérrezGutiérrez, T. Aguado, C. Sánchez-Ferrer, Inês J. Marques, M. Galardi-Castilla, I. de Diego, Manuel J. Gómez, A. Cortés, A. Zapata, Luis J. Jiménez-Borreguero, N. Mercader, I. Flores, Telomerase is essential for zebrafish heart regeneration, Cell Rep. 12 (2015) 1691-1703.

[18] A. Mosquera, J.L. Fernandez, A. Campos, V.J. Goyanes, J. Ramiro-Diaz, J. Gosalvez, Simultaneous decrease of telomere length and telomerase activity with ageing of human amniotic fluid cells, J. Med. Genet. 36 (1999) 494-496.

[19] W. Jia, S. Wang, J.W. Horner, N. Wang, H. Wang, E.J. Gunther, R.A. DePinho, J. Zhu, A BAC transgenic reporter recapitulates in vivo regulation of human telomerase reverse transcriptase in development and tumorigenesis, FASEB J. 25 (2011) 979-989.

[20] A. Gutkin, O. Uziel, E. Beery, J. Nordenberg, M. Pinchasi, H. Goldvaser, S. Henick, M. Goldberg, M. Lahav, Tumor cells derived exosomes contain hTERT mRNA and transform nonmalignant fibroblasts into telomerase positive cells, Oncotarget 7 (2016) 59173.

[21] J. Schindelin, C.T. Rueden, M.C. Hiner, K.W. Eliceiri, The Image ecosystem: an open platform for biomedical image analysis, Mol. Reprod. Dev. 82 (2015) 518-529.

[22] K. Suzuki, P. Bose, R.Y. Leong-Quong, D.J. Fujita, K. Riabowol, REAP: a two minute cell fractionation method, BMC Res. Notes 3 (2010) 294.

[23] T. Wu, C. Chen, S. Chen, Y. Xu, Y. Wang, Z. Chen, F. Wang, B. Xiao, Z. Tao, Nuclear translocation of telomerase reverse transcriptase is a critical process in lymphatic metastasis of nasopharyngeal carcinoma, Oncol. Lett. 9 (2015) 265-269.

[24] G. Di Noto, M. Chiarini, L. Paolini, E.L. Mazzoldi, V. Giustini, A. Radeghieri,
L. Caimi, D. Ricotta, Immunoglobulin free light chains and GAGs mediate multiple myeloma extracellular vesicles uptake and secondary NfiB nuclear translocation, Front. Immunol. 5 (2014) 517.

[25] D. Maiolo, L. Paolini, G. Di Noto, A. Zendrini, D. Berti, P. Bergese, D. Ricotta Colorimetric nanoplasmonic assay to determine purity and titrate extracellular vesicles, Anal. Chem. 87 (2015) 4168-4176.

[26] R.L. Tomlinson, T.D. Ziegler, T. Supakorndej, R.M. Terns, M.P. Terns, Cell cycleregulated trafficking of human telomerase to telomeres, Mol. Biol. Cell 17 (2006) 955-965.

[27] H. Seimiya, H. Sawada, Y. Muramatsu, M. Shimizu, K. Ohko, K. Yamane, $\mathrm{T}$. Tsuruo, Involvement of 14-3-3 proteins in nuclear localization of telomerase, EMBO J. 19 (2000) 2652-2661.

[28] H.K. Chung, C. Cheong, J. Song, H.W. Lee, Extratelomeric functions of telomerase, Curr. Mol. Med. 5 (2005) 233-241.

[29] Y. Zhang, X. Chen, X. Xu, X. Wang, X. Wang, G. Yuan, D. Sun, W. Ka, D. He Z. Wen, W. Yao, Knockdown of hTERT alters biophysical properties of K562 cells resulting in decreased migration rate in vitro, Cell Biochem. Biophys. 61 (2011) 595-603.

[30] G. Saretzki, Extra-telomeric functions of human telomerase: cancer, mitochondria and oxidative stress, Curr. Pharm. Des. 20 (2014) 6386-6403.

[31] L. Paolini, A. Zendrini, G. Di Noto, S. Busatto, E. Lottini, A. Radeghieri, A. Dossi, A. Caneschi, D. Ricotta, P. Bergese, Residual matrix from different separation techniques impacts exosome biological activity, Sci. Rep. 6 (2016) 23550.

[32] J.R. Edgar, Q\&A: what are exosomes, exactly? BMC Biol. 14 (2016) 46.

[33] H. Kalra, G. Drummen, S. Mathivanan, Focus on extracellular vesicles: introducing the next small big thing, Int. J. Mol. Sci. 17 (2016) 170. 